\title{
Current status and development trends of efficient oil production testing technology
}

\author{
Ning Kun ${ }^{1,2 *}$, Yu Gang ${ }^{3}$, Mu Lingyu ${ }^{1,2}, \mathrm{Wu} \mathrm{Xi}^{1,2}$ \\ ${ }^{1}$ CNPC Engineering and Technology R\&D Company Limited, Beijing, China \\ ${ }^{2}$ National Engineering Laboratory of Oil and Gas Drilling Technology, Beijing, China \\ ${ }^{3}$ Petroleum Engineering Research Institute of Dagang Oilfield, Tianjin 300280, China
}

\begin{abstract}
Oil production testing is a critical tool to understand reservoir properties and well producibility. The development of oil production testing technology in China has mainly gone through three phases: conventional oil production testing phase, oil production testing phase, and integrated oil production testing phase. Currently, domestic and overseas oilfields are still adopting the traditional oil production testing method, which contains plenty of repetitive processes that cost longer operating time, higher operating cost, formation damage caused by excessive high-density fluid, and many other shortcomings. Also, the difficulties of oil production testing in the deep and ultra-deep well under HPHT conditions are significant. Analyzing the development trend of oil production testing, there are two main topics identified: oil production testing for separated layer via one trip string and Pad-free oil production testing method. The oil production testing for separated layer via one trip string was developed for testing formations where lamination and thin layers are presented and causing interference among the testing layers. The Pad-free oil production testing methodology was developed for testing wells that fail to obtain reservoir fluid properties due to the formation damage caused by a high fluid density pad.

This paper is aiming to summarize and analyze the most advanced oil production testing technology worldwide and innovations had been made to the oil production testing field, hopefully inspired researchers to develop possible future uses and further improvements in efficiency. Facing the challenges of low gas prices, only continuous innovation in technology and the upgradation of tools and equipment can achieve the goal of improving quality and increasing efficiency.
\end{abstract}

\section{Introduction}

The history of oil testing in China began in the 1940s in Yumen (Laojunmiao), followed by the adoption of Soviet oil testing processes in the 1950s. And established of a formation testing research institute in Jianghan in the 1970s (in 1976, the South China Sea imported a Robley300 jack-up rig from Singapore, which came with a set of US Jonston 5" MFE testers). and the Ministry of Petroleum Industry set up a formation testing research institute in Jianghan, and started to organise translation, trials and research. 1978 saw the introduction of various types of formation testing tools from US companies such as Jonston MFE, Reiners PCT, Halliburton APR, Schlumberger HDT and Baker Hughes, as well as mapping and development. 1982 saw the first batch of 5" MFE testers produced by Baoji Petroleum Machinery Factory and Shanghai Dalong Petroleum Machinery Factory respectively. The first batch of 5" MFE test tools were copied and produced, and the first professional institution specialising in formation testing technology, introduction, research, promotion, training and technical services, the Oil and Gas Well Testing Centre, was established in Langfang in 1984. The centre has laid a solid foundation for the large-scale development of formation testing technology in China. Starting from 1987, testing institutions were established in oil fields throughout the country, beginning a whole new era of formation testing and oil testing techniques. At present, there are more than 120 professional testing teams of various types in China, capable of undertaking testing of various types, specifications and oil, gas and wells, with an annual working capacity of more than 3,000 test layers. The maximum well depth tested is 8,743 metres, the maximum hydrostatic column pressure is $151 \mathrm{MPa}$, the maximum downhole temperature is $216^{\circ} \mathrm{C}$.

With the emergence of high temperature and high pressure wells as well as deep wells, the exploration conditions and difficulties are becoming more and more difficult, and the requirements for the oil production testing process to obtain information on formation capacity and fluid properties are becoming higher and higher. Especially for the submerged mountain block, which the burial depth is shallow, fracture and cavity development, low pressure coefficient, easy to leak, complex lithology and other characteristics ${ }^{[1]}$. During construction operations, due to the geological characteristics of the submerged mountain reservoir, the

\footnotetext{
${ }^{a}$ Corresponding author: ningkundri@enpc.com.cn; Tel.: +86-15902227019
} 
reservoir is highly susceptible to contamination, so it is necessary to minimize the number of well killing and compressions during operations to avoid reservoir contamination to the greatest extent possible. The generalized oil production test operation has many drawbacks not only in terms of geology, but also in terms of engineering. This paper summarizes the process technologies that may become the future development trend of oil production testing by investigating the development and application of new technologies for oil production testing at home and abroad ${ }^{[2-5]}$. Include: "Oil Production Testing for Separated Layer via One Trip String" for test wells where there are interbedded layers and small layers that interfere with each other in terms of production, fluid properties and pressure; the "No Pad Oil Testing Technology" for test wells where there is contamination of the test pad and difficult to get the reservoir fluid. Faced with the advent of the low oil price era, only continuous innovation in technology and upgrading of tools and equipment can achieve the goal of improving quality and increasing efficiency, and refined oil testing will become an important development direction in the future.

\section{Oil production testing for separated layer in long well section}

\subsection{Testing string construction}

The long section oil production testing string for separated layer consists of heavy duty screen pipe, mark, mechanical firing head, TCP gun, RTTS packer, pressure controlled mechanical switching valve, vertical shock absorber, hydraulic firing head and memory gauge (Figure 1), connected in sequence from top to bottom.

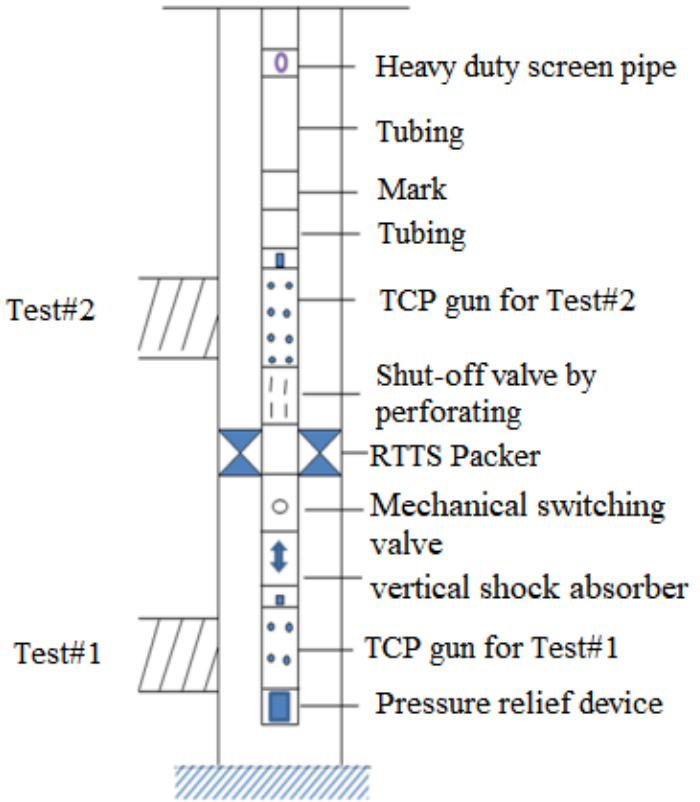

Figure 1 Schematic diagram of the fine layered test oil string

In the above testing string, the heavy duty screen pipe is provided with side holes, mounted on the upper part of the mark, to allow connect tubing with casing and provide a flow path for the perforated formation. The mark is located between the heavy duty screen pipe and the second layer TCP, and acts as a depth correction. Firing the second layer TCP by drop a bar. The purpose of the screen pipe is to transfer pressure and form the oil flow path of the first test layer; the sand bowl is connected to the second test layer and the lower part of the screen pipe to prevent the sand of the second test layer from burying the packer and prevent the packer from unsealing properly. The RTTS packer is installed between the two test intervals to isolate, avoiding mutual interference between the two layers in terms of pressure, liquidity and other geological information, thus serving the purpose of layered oil testing. The lower part of the packer is connected to a pressure-controlled mechanical valve, which is opened by the wellhead pressurisation and pressure release. The shock absorber is used to release the axial vibration to the testing string during perforation. The first TCP is fired through the annulus pressure. There are memory gauges connected below the TCP for the built-in pressure gauge, which records the pressure information during the test

\subsection{Process principles}

Fine layered oil production testing technology is a new concept that is different from the general oil production testing technology. It is different from the conventional oil production testing practice of "shooting one layer, testing one layer, blocking one layer" or shooting multiple layers together. Fine layered oil production testing refers to one trip testing string carrying two or more sets of TCP guns, packers and pressure-controlled mechanical switches and other devices, which can achieve two or more test layers of independent oil production testing operations, so as to achieve the effect that the production data will not interfere with each other. The ultimate goal is to shorten operation time, save operation cost and reduce labour intensity ${ }^{[6]}$.

The operation procedure is as follows: RIH the string into the well, fired the TCP after correction the depth of gun, the RTTS packer is seated in the casing by lifting the string and lowering to achieve the purpose of sealing the upper and lower test layers. Through the annular pressure between the tubing and casing, the pressure is transmitted through the screen pipe to the first layer of the firing head, and the pressure fired the gun. The formation fluid upward through the side holes of the screen pipe and the heavy screen pipe and tubing eventually flow to the surface. After the first test layer is released and sprayed for production, the annulus is pressurised to close the pressure-controlled mechanical valve, and the liquid nitrogen is discharged to reduce the wellbore level so that the second layer forms a negative pressure during perforation operation with drop a bar. When the second test layer has been tested, it is possible to decide whether to open the switch valve for a combined test of the two layers under pressure in the annulus according to the needs of the site. After completion of the test, circulate the well until the well is stable, and then release the RTTS packer by lifting the string and pull out of the well. 


\subsection{Example analysis}

The oil production testing in separated layer was first applied in a block 1601 well in Dagang oilfield, and the operation work and oil production test results achieved satisfactory results. The well is a exploration well in the Dagang oilfield, with a completion depth of $4437 \mathrm{~m}$ and a completion formation of Sha Yi Zhong, designed to test two layers of oil, with the test formation being Plate 0 . The well is expected to test at a formation temperature of around $100^{\circ} \mathrm{C}$. Considering the safety and reliability of the operation, the TCP gun powder type was preferred, with Hesokin RDX lasting nearly 10 days and Otokin HMX lasting nearly 25 days. The results of the TCP gun preference are shown in the table below.

\begin{tabular}{|c|c|c|c|c|c|c|c|}
\hline & Charge & $\begin{array}{c}\text { Type of } \\
\text { powder }\end{array}$ & $\begin{array}{c}\text { Temperature } \\
\text { resistance } \\
\left({ }^{\circ} \mathrm{C}\right)\end{array}$ & $\begin{array}{c}\text { Shoots } \\
(\text { Holes/m) }\end{array}$ & $\begin{array}{c}\text { Phases } \\
\left({ }^{\circ}\right)\end{array}$ & $\begin{array}{c}\text { Hole } \\
\text { diameter } \\
(\mathrm{mm})\end{array}$ & $\begin{array}{c}\text { Depth of } \\
\text { penetration (mm) }\end{array}$ \\
\hline 89 & 89 & HMX & $100 / 25$ days & 16 & 90 & 8.8 & 451 \\
\hline
\end{tabular}

\subsection{Accounting for cost savings}

According to the Dagang oilfield operating cost quotations, the well was tested in layers via one trip string, which reduced the procedures of RIH \& $\mathrm{POOH}$ string, killing the well in circulation, filling and sealing the sand and disassembling the wellhead, etc.; the operating time was shortened by 7.8 days and the operating cost was saved by more than RMB 410,000.

\subsection{Oil production testing results}

The well operation went smoothly. The well had a significant independent oil production test result, the first test layer obtained a daily production of 31.3 square meters, formation pressure $28.35 \mathrm{MPa}$, pressure coefficient 1.1, formation temperature $97.2^{\circ} \mathrm{C}$, temperature gradient $3.7^{\circ} \mathrm{C} / 100 \mathrm{~m}$, the test oil conclusion was oil formation. The second test layer obtained a daily production of 8.52 square meters, formation pressure $28.18 \mathrm{MPa}$, pressure coefficient 1.1, formation temperature $96.6^{\circ} \mathrm{C}$, temperature gradient $3.7^{\circ} \mathrm{C} / 100 \mathrm{~m}$, the oil production test conclusion is oil formation.

\section{Oil production testing technique without liquid pad}

\subsection{Testing string construction}

The oil production test string consists of mark, input throttle valve, memory gauge, packer, vertical shock absorber, opening device and TCP gun (Figure 2), which are connected in sequence from top to bottom. The input throttle valve is the key tool in this string, through its throttling effect, to control the test pressure difference, to achieve less liquid cushion or even no liquid cushion oil production test operation.

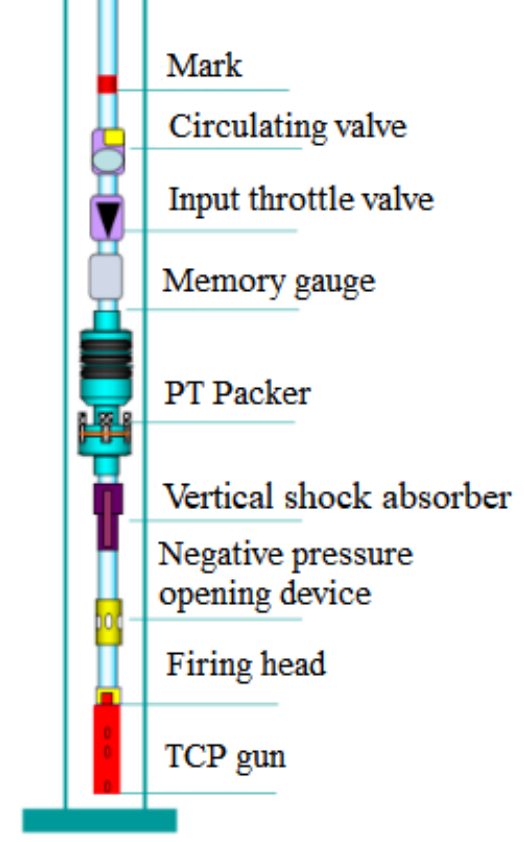

Figure 2 Schematic diagram of the oil production testing string without liquid pad

\subsection{Process principles}

During current oil production testing operations, fluid pads are usually added to the wellbore, including water, diesel, inorganic and organic salts. The main purpose of adding fluid pads is to control the differential pressure of the formation production, so that the fluid can achieve negative pressure to induce spraying and unblock without destroying the equilibrium of the formation, and also to protect the casing from damage and the packer from seepage. However, there are problems of high drainage costs and difficulty in determining the fluid properties of the formation.

The string consists of perforation section and test section, where the perforation section consists of an annular pressure stimulator, packer, shock absorber and perforated gun, and the test section consists of test 
intervals and formation pressure balance sections. The self-controlled throttle valve is directly connected to the string through threads at the top and bottom, which can automatically adjust the throttle outlet size according to the formation pressure, thereby controlling the production pressure differential within a reasonable range. This technology will replace the previous process of balancing the production pressure differential with a liquid pad, eliminating the need for the design and operation of liquid pad, achieving more efficient and economical testing of production and avoiding damage to the formation due to excessive production pressure differential.

After perforated, the tester is opened and recorded the whole process of pressure change. Due to the automatic adjustment of the differential pressure by the flow adjust valve, the tester will not be affected by the large differential pressure for a long time during the whole testing process, which will not cause a large amount of sand out of the formation and affect the production capacity and the accuracy of the test. As the test is conducted without a liquid cushion, no external fluid enters the tester at the moment of opening, and the fluid obtained from the test sampling is all formation fluid.

\subsection{On-site applications}

The oil production test layer of X1601 well in Dagang oilfield: 1425.9-1442.6m and 1470.6-1482.2m was tested in the field for oil production test technology without liquid cushion. After completing the process of drifting, scraping and bubbling water to lower the liquid level (the liquid cushion was $5 \mathrm{~m}$ below the throttle), drop a bar with open well state, opened the negative pressure opening device after 30 seconds, and delayed opening for production and no oil or gas showing. A total of five water samples were obtained from the well wash outlet and compared with the results of water samples from the same and adjacent layers of the neighbouring wells, the water was basically satisfactory.

\section{Conclusion}

(1) The split-layer oil production testing process can reduce field work procedures, shorten operation time and lower operating costs; compared with conventional single-layer oil production testing, it saves more than RMB 410,000 in testing costs and shortens the testing period by 7.8 days. The technology has been applied to more than ten wells in Dagang Oilfield, all of which have been tested for two layers via one trip string and achieved good results.

(2) The liquid cushion-free oil production testing technology achieves control of the test pressure difference through the down hole throttling valve, avoiding the liquid cushion's interference with the formation liquidity, which is of great significance to the exploration and evaluation of low-permeability reservoirs.

(3) Both of the above-mentioned oil production testing process technologies, through the combination of geology and engineering, can meet the geological and engineering requirements to the greatest extent, and have broad market promotion and application prospects.

\section{References}

1. WU Yongping, YANG Chiyin, FU Lixin, XIAO Dunqing, MA Xiaoming. Exploration and discovery of condensate fields in the Bohai Bay Basin [J]. Offshore Oil and Gas Geology,2007(03):44-52.

2. Zhang Shaoli. The formation and development direction of oil production testing technology[J]. Oil and Gas Well Testing,2003(06):63-70.

3. Zhang X. Analysis of the current situation and trend of oil production testing process application in low permeability reservoirs[J]. Chemical Management,2018(06):116.

4. Zhou Min. Exploration of oil production testing process and development trend $[\mathrm{J}]$. Science and Technology Outlook,2016,26(19):92.

5. Wang Jingwu. Research on the application development of oil production testing process technology[J]. Modern Trade Industry,2009,21(11):298-299.

6. MA Jinliang, LIU Zeyu, LI Chunning, HE Xinwei, LEI Guoyan. One-trip tubular column stratified injection and hydraulic pumping fluid discharge coworking technology[J]. Oil and gas well testing,2018,27(02):22-26. 